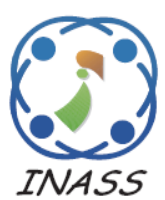

http://www.inass.org/

\title{
Image Encryption using Double Layer Chaos with Dynamic Iteration and Rotation Pattern
}

\author{
Fikri Budiman $^{1 *}$ \\ Pulung Nurtantio Andono ${ }^{2}$ \\ De Rosal Ignatius Moses Setiadi ${ }^{2}$ \\ ${ }^{1}$ Faculty of Computer Science, Dian Nuswantoro University, Semarang, Indonesia \\ ${ }^{2}$ Department of Informatics Engineering, Dian Nuswantoro University, Semarang, Indonesia \\ * Corresponding author's Email: fikri.budiman@dsn.dinus.ac.id
}

\begin{abstract}
This research proposes an encryption method for images based on dual chaos systems and dual hash functions. SHA-256 and SHA-512 are hash functions chosen to process plain images and private keys. The purpose of using hash functions on plain and key images is to increase encryption resistance against differential attacks and perform local encryption in each image zone. The proposed method consists of two stages of encryption, the first involves dual hash functions and confusion with a chaotic system by performing encryption based on zoning and rotation of the image. The second stage is a diffuse process using a logistic map. The proposed method is proven to be strong against various attacks that have been tested with various measuring tools such as entropy, histogram analysis, chi-square, number of changing pixel rate (NPCR), unified averaged changed intensity (UACI), and the avalanche effect (AE). This method also proved to work better than the state-of-the-art method in this research, significantly based on entropy, UACI, and NPCR.
\end{abstract}

Keywords: Chaotic system, Logistic map, Zoning and rotate, Cryptosystem.

\section{Introduction}

Technology is growing and computer computing is getting faster, this has many positive benefits for humans in facilitating their work, for example in data transmission. However, this can also be a problem when used by irresponsible people and commit cybercrimes. Therefore, the security of data transmission needs to be strengthened [1]. Encryption is one way of securing data and is currently one of the interesting studies to study. Actually all digital data can be encrypted, but encryption research on image data has become the most conducted [2, 3]. Encryption in digital images has different characteristics when compared to text encryption. Several methods for text encryption, such as Advanced Encryption Standard (AES), Data Encryption Standard (DES), 3-DES, and RivestShamir-Adleman (RSA), have been proven to work well on text, but if applied to encryption of images or multimedia data, they are not suitable. Many researchers, such as in research [4-8], state that these methods make the encryption process less efficient, over-complex, and over-computing. Encryption on images is widely applied using the chaos method, which this method has many advantages in encrypting a digital image, some of which are ergodicity characters, sensitivity to more unpredictable initial values, low complexity, and strong correlation, and non-uniform distribution. [911], this is the reason for choosing the chaos system in this research.

There are many chaotic systems used in image encryption, such as Arnold [5, 7], logistic map [1215], hyperchaotic [16, 17], and others. In many studies of encryption literature on digital images, the use of chaos systems is widely combined with various other encryption methods to improve message security. But if you look specifically, some research proposes a block-based encryption technique based on a chaos system as in the research $[3,18]$. The block-based technique is done by dividing the image into smaller blocks, then encrypting each block. In research conducted by [3], Encryption of each block 
is done dynamically with a different key and is also done with a dynamic pattern, so that each image block will indirectly have an independent key and increase security.

Another quiet method that is widely combined with the chaotic method is hashing. Hashing is a oneway encryption method that is widely used to encrypt passwords because the results of hashing encryption cannot be decrypted. The hashing method is implemented in image encryption, usually in the add key generation process. The key in question is generally a key that is processed from plaintext [10, 19-21] as well as the private key used for encryption [3], In addition, the hash method is also widely used to process keys generated from pseudo-random generators [17]. The purpose of the hash function here can serve to increase security from differential attacks because with the help of the hash function it can increase the diversity of keys when there is a minimal modification of the plaintext. Based on these hypotheses, this study develops an encryption method based on a chaotic algorithm combined with a hash function, then this research provides several modifications to the model for combining the chaos method with zoning and rotating techniques. This paper is composed of six parts, namely an introduction to this section, part two explaining related research, part three explaining the proposed model, part four presenting the results and analysis, part five presenting the discussion, and part six containing a conclusion.

\section{Related research}

Several studies have inspired this research in modifying the encryption model, one of which is research [7]. In this research, the encryption method is carried out by combining the chaotic method and the hybrid modulus substitution cipher, both of which are encryption with a chaotic system. The chaotic method is used to perform shuffle and hybrid modulus substitution cipher is used for the diffusion process.

Other research is [22], In this research, the contribution is in the 1-shaped method of dynamic block. This method also uses a chaotic method combined with a hash function (SHA 512). SHA-512 is used to generate keys from plain images. In the early stages of encryption, each dynamic block is carried out with an L-shaped pattern to perform the scrambling process. The next step is the diffusion process with the 1 shape pattern and XOR function.

In research [3], the encryption technique is divided into stages, the first is confusion with the Arnold method locally in each sub-block then followed by a diffusion technique using a stream cipher. By using both stages of encryption, encryption is produced that is resistant to statistical and differential attacks.

Research [23] proposes a two-stage encryption technique, where the first is a horizontal and vertical permutation process. The permutation technique is similar to the confusion and scrambling technique, the goal is to randomize the position of the image pixels. The permutation process is carried out based on a random keystream generator. While the diffusion process is carried out based on a dynamic index generator. The diffusion process is carried out simultaneously based on certain iterations to produce an encrypted image.

From the several methods described, it can be concluded that the image encryption process must have at least two processes, namely confusion/permutation/scrambling and the diffusion stage. By combining these two processes will produce a strong encryption method. Tetapi proses enkripsi banyak dilakukan langsung pada whole image. Inspired by [3], This research also uses these two processes to propose an encryption model, to increase resistance to statistical attacks, a slight modification was carried out with a gradual encryption method based on local confusion with zoning and rotation techniques in the early stages, while at the other stage XOR operations were carried out with a logistic map. Furthermore, the hash function is performed on two of the key and on the image to increase the security of the differential, this method is further explained in section 3 .

\section{Proposed method}

In this section, a symmetric encryption method is proposed which involves several methods with modifications in several parts. The proposed method consists of two main processes, namely encryption, and decryption. These two processes involve two chaotic methods, hash functions combined with zoning and rotation techniques. In detail the encryption process is explained as follows:

1. Read the plain image, use it as input for two processes, namely: the SHA-1 hash function and the zoning function. As a limitation, the plain image used has a size of $512 \times 512$ pixels.

2. SHA-256 hash function of plain image $(P)$ will generate $k e y B$ as the key to performing the rotation. Also, perform the SHA-256 hash function for the complement value of the image $\left(P^{\prime}\right)$. The results of the two SHA functions are combined and produce a 64-character keyB key. For an illustration see Eq. (1) create keyB. 


$$
\operatorname{keyB}=\operatorname{JOINT}\left(\operatorname{SHA256}(P), \operatorname{SHA256}\left(P^{\prime}\right)\right)
$$

3. Divide the image into small zones with a size of $64 \times 64$. So, the image is divided into 64 zones.

4. Change each keyB character to ASCII number form, resulting in 64 ASCII numbers for 64 zones. Then perform the modulus 4 function on each character to determine the rotation angle of each zone.

5. Rotate the zone based on the remaining ASCII quotient $($ key $B)$. For the remaining 0 no rotation is performed, the remaining 1 is rotated $90^{\circ}$, the remaining 2 is rotated $180^{\circ}$, the remaining 3 is rotated 270, see Fig. 1 for sample results. Save key $B$ for the decryption process.

6. On the other hand, read keyA then encrypt the SHA512 hash, then convert the encryption result to an ASCII number

7. Perform the modulus operation for each ASCII number to get the chaotic encryption key for each image zone. Use Eq. (2) to get the $p$ value, Eq. (3) to get the value of $q$, Eq. (4) for the number of iterations $(r)$, and Eq. (5) to perform a chaotic operation. So that the chaotic operation is carried out dynamically on each image zone based on keyA.

$$
\begin{array}{r}
p=\bmod (\operatorname{ASCII}[i], 256) \\
q=256-\bmod (\operatorname{ASCII}[i], 256) \\
r=\bmod (\operatorname{ASCII}[i], 32) \\
{\left[\begin{array}{l}
x^{\prime} \\
y^{\prime}
\end{array}\right]=\left[\begin{array}{cc}
1 & p \\
q & p q+1
\end{array}\right]\left[\begin{array}{l}
x \\
y
\end{array}\right] \bmod M}
\end{array}
$$

8. Where the image must have a size of $M \times M, x$ and $y$ are the pixel coordinates of the original image, $x^{\prime}$ and $y^{\prime}$ are the pixel coordinates of the image after scrambled, the chaotic operation is carried out with the number of iterations $r, i$ is the index of each ASCII number. The results of this stage will produce the first stage of encryption.

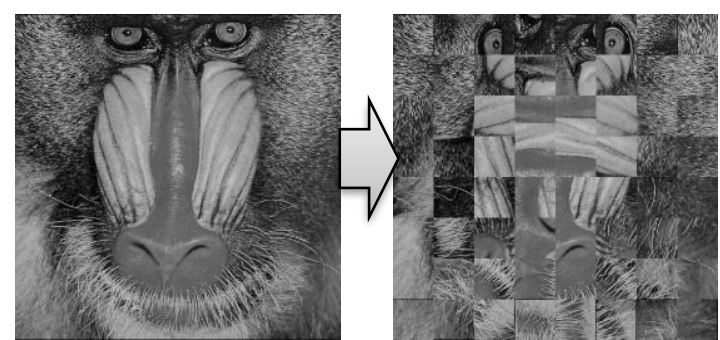

Figure. 1 Sample result after rotate zoning image

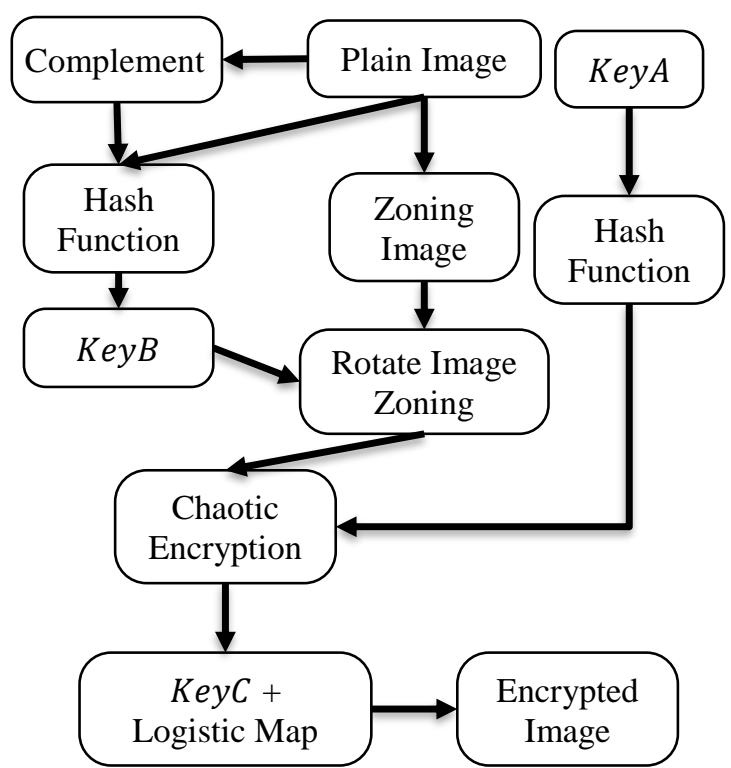

Figure. 2 Encryption Model

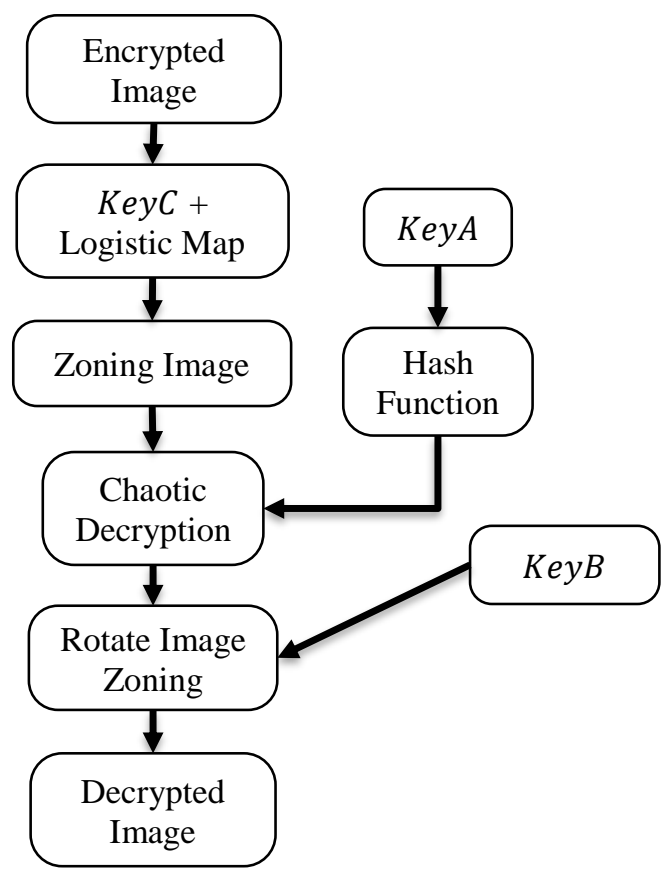

Figure. 3 Decryption model

9. Create $L$ with a logistic map with Eq. (6).

$L_{n+1}=\operatorname{round}\left(\right.$ keyC $\left.L_{n}\left(1-L_{n}\right) \times 255\right)$

Where $L_{0}$ is the initial value, $0<L_{0}<1$, $k e y C$ is the bifurcation parameter $0<$ keyC $<4$, selected keyC $=3.99$, while $n=262144$ is the logistic length.

10. Reshape the first stage of encryption into an array and then perform the XOR operation with $L$.

11. Get the encrypted image by reshaping the encrypted array. 
To see in more detail all the encryption steps, see Fig. 2. At the decryption stage, it is explained in detail as follows:

1. Use the encrypted image as the first input, then reshape the encrypted image into an array.

2. Perform the 8th step in the encryption process, to create an array logistic map.

3. Perform XOR operation on $L$ and array encrypted image. Then reshape the results of the XOR operation into an image with a size of $M \times M$.

4. Do zoning image.

5. Enter KeyA, then perform the SHA512 hash operation. Then convert to ASCII numbers. To get the values of $p, q$ and $r$ use Eq. (2), Eq. (3), and Eq. (4).

6. Perform chaotic decryption on each image zone based on the values of $p, q$ and $r$ using Eq. (7).

$$
\left[\begin{array}{l}
x \\
y
\end{array}\right]=\left[\begin{array}{cc}
1 & p \\
q & p q+1
\end{array}\right]^{-1}\left[\begin{array}{l}
x^{\prime} \\
y^{\prime}
\end{array}\right] \bmod M
$$

7. Enter $K e y B$ to derotate each decrypted image zone so that a complete decrypted image is obtained.

For more clearly the method of decryption can be seen in Fig. 3. For the note, the above process is carried out for grayscale images. In RGB images, the same process is carried out on each color channel.

\section{Results and analysis}

At this stage the proposed encryption model is tested on a public dataset that has been applied to previous research, the aim is to find out how much contribution is generated. The image dataset used is presented in Fig. 4. The image dataset used consists of 13 images, all of which have dimensions of $512 \times 512$ pixels, where 10 images are standard images with two types of models, namely grayscale and RGB, while the other three images are batik images. Batik is one of the original cultural heritage of Indonesia. This batik image is used in this study because in this research it is one of the research branches created by the author in developing Batik research, which has previously been published in research $[24,25]$. The goal is to develop the security of storing batik images. Furthermore, the encryption model is implemented on each image so that the resulting encrypted image is presented in Fig. 5.

Visually the results presented in Fig. 5 have a broken display and have no correlation with the original image, this means that the resulting encryption is good. However, the visual display does
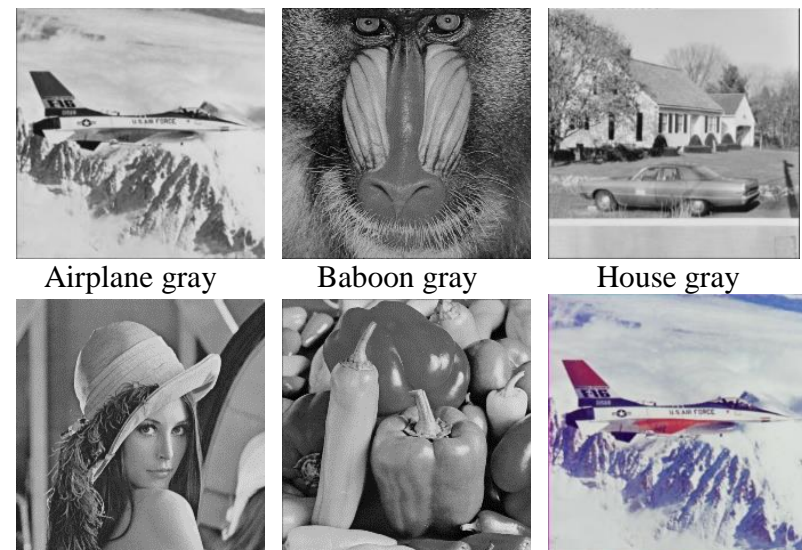

Baboon gray

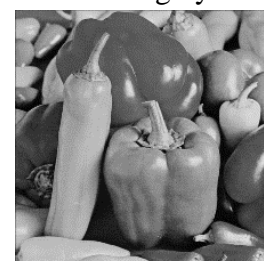

House gray

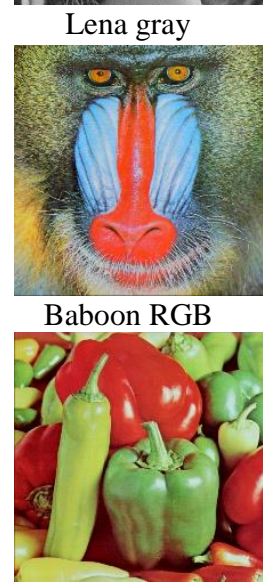

Peppers RGB
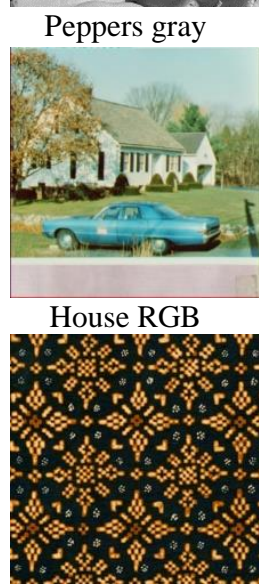

Batik Nitik

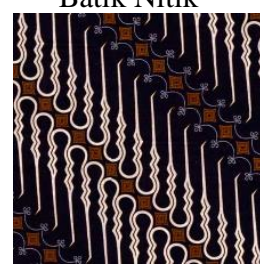

Batik Parang

Figure. 4 Image dataset

not have a definite value so that more measurable measurements are needed. Therefore, several measures are used, the first is information entropy.

Information entropy is a measuring tool used to calculate the randomness value of an image. The encrypted image should have a greater entropy value than the original image. The maximum value of entropy is 8 , and if the entropy of the encrypted image has a value that is getting closer to 8 , it means that the encryption results based on entropy are very good.[26]. The entropy value can be calculated by Eq. (8), while the calculation results are presented in Table 1.

$$
H_{i}=-\sum_{0}^{2^{8}-1} p_{i} \log _{2}\left(p_{i}\right)
$$

Where $H_{i}$ is entropy, $p_{i}$ and $\log _{2} p_{i}$ refer to the 

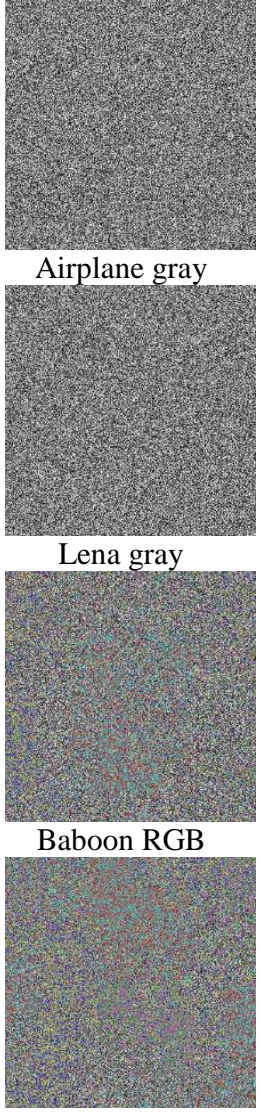

Peppers RGB
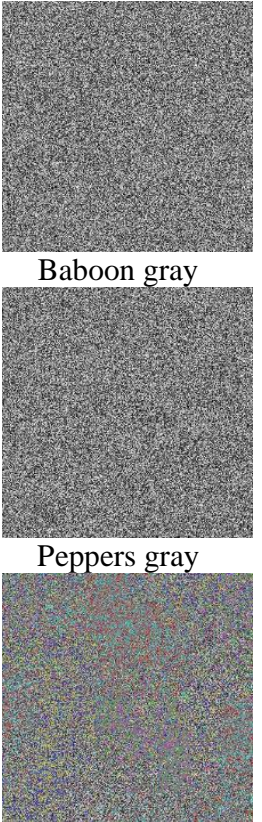

House RGB

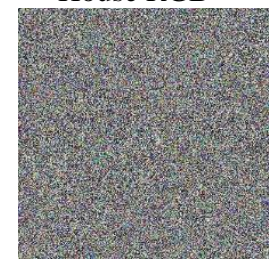

Batik Nitik

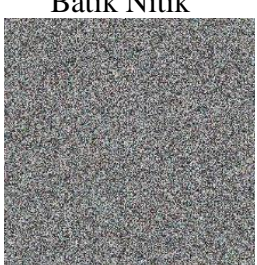

Batik Parang

Figure. 5 Encrypted image results

Table 1. Entropy results

\begin{tabular}{|c|c|c|}
\hline \multirow{2}{*}{ Image } & \multicolumn{2}{|c|}{ Entropy } \\
\cline { 2 - 3 } & $\begin{array}{c}\text { Before } \\
\text { Encryption }\end{array}$ & $\begin{array}{c}\text { After } \\
\text { Encryption }\end{array}$ \\
\hline Airplane gray & 6.7025 & 7.9997 \\
\hline Baboon gray & 7.3771 & 7.9996 \\
\hline House gray & 7.2334 & 7.9995 \\
\hline Lena gray & 6.8677 & 7.9998 \\
\hline Peppers gray & 7.5937 & 7.9997 \\
\hline Airplane RGB & 6.6639 & 7.9997 \\
\hline Baboon RGB & 7.7624 & 7.9996 \\
\hline House RGB & 7.4858 & 7.9997 \\
\hline Lena RGB & 7.7502 & 7.9998 \\
\hline Peppers RGB & 7.6698 & 7.9998 \\
\hline Batik Kawung & 7.4148 & 7.9997 \\
\hline Batik Nitik & 7.0165 & 7.9996 \\
\hline Batik Parang & 6.6716 & 7.9997 \\
\hline Average & 7.2469 & 7.9997 \\
\hline
\end{tabular}

Table 2. Entropy comparison in lena color image

\begin{tabular}{|l|c|}
\hline \multicolumn{1}{|c|}{ Approach } & Entropy \\
\hline Research [27] & 7.9993 \\
\hline Research [28] & 7.9993 \\
\hline Research [29] & 7.6635 \\
\hline Research [3] & 7.9997 \\
\hline Proposed & 7.9998 \\
\hline
\end{tabular}

Table 3. Entropy comparison in lena gray image

\begin{tabular}{|l|c|}
\hline \multicolumn{1}{|c|}{ Method in } & Entropy \\
\hline Research [30] & 7.9972 \\
\hline Research [5] & 7.9976 \\
\hline Research [31] & 7.9993 \\
\hline Research [3] & 7.9993 \\
\hline Proposed & 7.9998 \\
\hline
\end{tabular}

probability of the occurrence of the symbol $i$ and base 2 logarithms, respectively

Based on the results presented in Table 1, it appears that there is a significant increase in the entropy results. The entropy value is very close to 8 , this means that the entropy-based image encryption security is proven to be good. In addition, this study also carried out several comparisons with other related methods, the comparison of entropy is presented in Table 2.

The second test was performed using NPCR and UACI values. Both of these measuring tools serve to measure encryption resistance against differential attacks. Both of these measuring instruments can be used by using two encrypted images (E1 and E2) which are generated by replacing the 1-bit plain image. NPCR stands for the number of pixels change rate which can be calculated by Eq. (9), while UACI stands for Unified Average Changing Intensity which can be calculated by Eq. (10) [32]. The ideal value of NPCR is 99.6093 and UACI is 33.4635 [33]. The results of the NPCR and UACI measurements are presented in Table 4.

$$
\begin{gathered}
N P C R=\left(\frac{1}{M \times N} \sum_{x=0}^{M} \sum_{y=0}^{N} D(x, y)\right) \\
D(x, y)=\left\{\begin{array}{l}
0, E_{1}(x, y)=E_{2}(x, y) \\
1, \text { otherwise }
\end{array}\right. \\
U A C I=\left(\frac{1}{M \times N} \sum_{x=0}^{M} \sum_{y=0}^{N} \frac{\left|E_{1}(x, y)-E_{2}(x, y)\right|}{\left(2^{8}\right)-1}\right)
\end{gathered}
$$

For the record, the NPCR and UACI values 
Table 4. UACI and NPCR results

\begin{tabular}{|c|c|c|}
\hline Image & UACI & NPCR \\
\hline Airplane gray & 33.5114 & 99.5682 \\
\hline Baboon gray & 33.4192 & 99.6168 \\
\hline House gray & 33.4331 & 99.5897 \\
\hline Lena gray & 33.4517 & 99.5904 \\
\hline Peppers gray & 33.5251 & 99.6487 \\
\hline Airplane RGB & 33.3909 & 99.6234 \\
\hline Baboon RGB & 33.4287 & 99.5979 \\
\hline House RGB & 33.4321 & 99.6368 \\
\hline Lena RGB & 33.5098 & 99.6016 \\
\hline Peppers RGB & 33.4097 & 99.5897 \\
\hline Batik Kawung & 33.4354 & 99.5946 \\
\hline Batik Nitik & 33.4134 & 99.6319 \\
\hline Batik Parang & 33.4314 & 99.6213 \\
\hline Average & 33.4455 & 99.6085 \\
\hline
\end{tabular}

Table 5. UACI and NPCR comparison in lena color image

\begin{tabular}{|l|c|c|}
\hline \multicolumn{1}{|c|}{ Approach } & UACI & NPCR \\
\hline Research [27] & 33.2800 & 99.6000 \\
\hline Research [28] & 33.4707 & 99.6098 \\
\hline Research [29] & 33.4254 & 99.6082 \\
\hline Research [3] & 33.2349 & 99.6009 \\
\hline Proposed & 33.5098 & 99.6016 \\
\hline
\end{tabular}

Table 6. UACI and NPCR comparison in lena gray image

\begin{tabular}{|l|r|c|}
\hline \multicolumn{1}{|c|}{ Approach } & UACI & NPCR \\
\hline Research [34] & 33.2161 & 99.4602 \\
\hline Research [5] & 28.6600 & 99.6400 \\
\hline Research [31] & 33.4197 & 99.6213 \\
\hline Research [3] & 33.4192 & 99.6212 \\
\hline Proposed & 33.4517 & 99.5904 \\
\hline
\end{tabular}

presented on the RGB image are the average values of the R, G, and B layers in each image, while the plaintext modification of the image is located at location 256,256 .

Our third test measures the avalanche effect (AE). $\mathrm{AE}$ is also a measuring tool to determine the resilience of differentials such as NPCR and UACI, the difference is that $\mathrm{AE}$ tends to use small modifications (usually 1-bit) in the password, then compared to two encrypted images (E1 and E2). In this method, modifications are made to keyA (see Fig. 2. AE can be calculated with Eq. (11), where the ideal $\mathrm{AE}$ value is $50 \%$. In this study, the $\mathrm{AE}$ value was calculated in three trials, where modifications of the 1-bit password were performed at the front, middle, and end. AE test results are presented in
Table 7. Avalanche effect results

\begin{tabular}{|l|c|c|c|}
\hline \multirow{2}{*}{ Image } & \multicolumn{3}{|c|}{ Bit Password Modification in (\%) } \\
\cline { 2 - 4 } & Initial & Middle & Last \\
\hline Airplane gray & 50.0109 & 50.0034 & 50.0311 \\
\hline Baboon gray & 50.0192 & 50.0426 & 50.0415 \\
\hline House gray & 50.0139 & 50.1075 & 50.0438 \\
\hline Lena gray & 50.0172 & 50.0213 & 50.0091 \\
\hline Peppers gray & 50.0560 & 50.0228 & 50.0239 \\
\hline Airplane RGB & 50.0312 & 49.9950 & 50.0118 \\
\hline Baboon RGB & 49.9892 & 50.0002 & 50.0104 \\
\hline House RGB & 50.0514 & 50.0241 & 50.0023 \\
\hline Lena RGB & 50.0018 & 50.0103 & 50.0114 \\
\hline Peppers RGB & 49.9985 & 50.0391 & 49.9986 \\
\hline Batik Kawung & 50.0053 & 49.9981 & 50.0218 \\
\hline Batik Nitik & 50.0108 & 50.0396 & 50.0275 \\
\hline Batik Parang & 50.0518 & 50.0057 & 49.9892 \\
\hline Average & 50.0198 & 50.0238 & 50.0171 \\
\hline Average all & \multicolumn{4}{|l}{50.0202} \\
\hline
\end{tabular}

Table 8. Avalanche effect comparison in lena image

\begin{tabular}{|l|l|l|}
\hline \multicolumn{1}{|c|}{ Approach } & \multicolumn{1}{|c|}{ Image Type } & \multicolumn{1}{c|}{$\begin{array}{c}\text { Avalanche } \\
\text { Effect (\%) }\end{array}$} \\
\hline Research [35] & Color & 50.0123 \\
\hline Research [3] & Color & 49.9822 \\
\hline Proposed & Color & 50.0159 \\
\hline Research [5] & Gray & 49.9767 \\
\hline Research [3] & Gray & 50.0366 \\
\hline Proposed & Gray & 50.0078 \\
\hline
\end{tabular}

$$
\begin{gathered}
A E=\frac{\sum_{x=1}^{M} \sum_{y=1}^{N} D_{x, y}}{M \times N} \times 100 \%, \\
D_{x, y}\left\{\begin{array}{l}
E_{1}(x, y) \neq E_{2}(x, y), 1 \\
E_{1}(x, y)=E_{2}(x, y), 0
\end{array}\right.
\end{gathered}
$$

Table 7.

The fourth test is carried out histogram analysis and chi-square test, the aim is to determine the encryption resistance of statistical attacks [6]. Visually, the histogram that looks uniform and has a significant difference from the original image histogram indicates that the quality of the proposed encryption is very good. Histogram results from image encryption are presented in Fig. 6. Even though the chi-square $\left(\chi^{2}\right)$ measurement used to validate this quality. The value of chi-square can be calculated by Eq. (12), and the results of the calculations are presented in Table 9.

$$
\chi^{2}=\sum_{x=1}^{i} \frac{\left(f_{x}-g_{x}\right)^{2}}{g_{x}}
$$



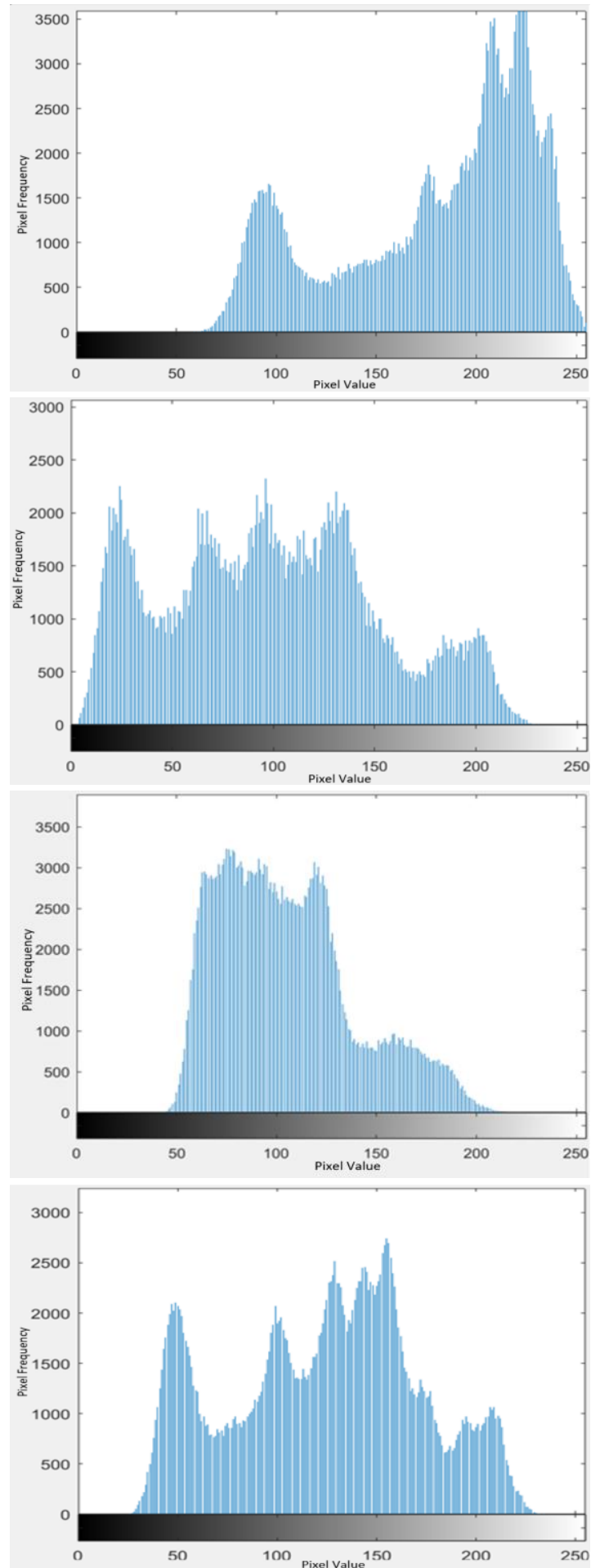
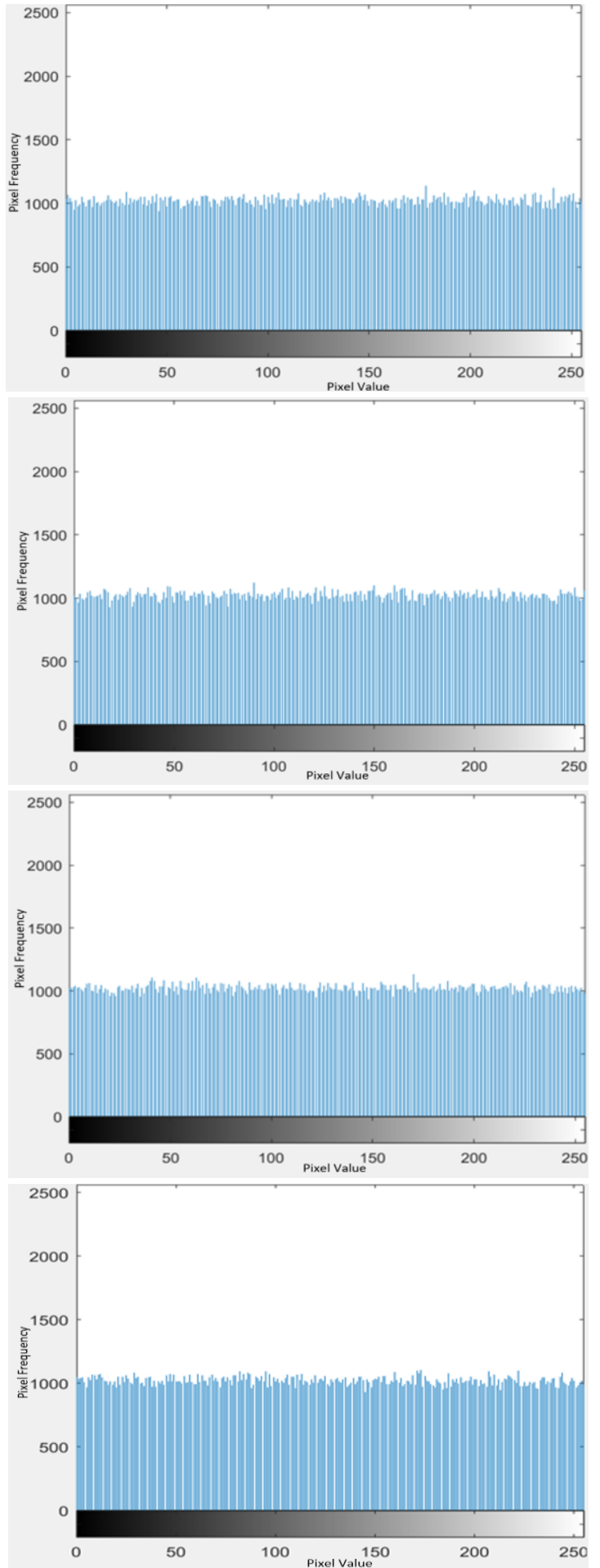

Figure. 6 Sample histogram of Lena image, the first column is the original histogram, the second column is an encrypted histogram, the first row is red channel, the second row is green channel, the third row is the blue channel, the fourth row is the gray channel

Where $i$ is gray-level, $g_{x}$ is the pixel frequency of each gray value to the theoretical histogram, $f_{x}$ is the pixel frequency of each gray value concerning the actual histogram, $\chi^{2}$ represents the degree of deviation between the tested histogram and the ideal histogram. When the significant level is 0.05 , then $\chi_{0.05}^{2}(255)=293.24783$, so when the value of $\chi^{2}$ encrypted image $<\chi_{0.05}^{2}$ can be concluded the histogram is approximately uniform.

Based on the results presented in Table 9, it appears that 10 of the 13 images have the ideal chi- 
Table 9. Chi-square results

\begin{tabular}{|c|c|}
\hline Image & $\boldsymbol{\chi}^{\mathbf{2}}$ \\
\hline Airplane gray & $\mathbf{3 1 5 . 7 2 8 8}$ \\
\hline Baboon gray & 286.3879 \\
\hline House gray & 277.9237 \\
\hline Lena gray & 289.7390 \\
\hline Peppers gray & $\mathbf{3 1 0 . 3 8 2 9}$ \\
\hline Airplane RGB & 257.1270 \\
\hline Baboon RGB & 286.8623 \\
\hline House RGB & $\mathbf{2 9 8 . 8 9 1 3}$ \\
\hline Lena RGB & 291.2394 \\
\hline Peppers RGB & 283.3699 \\
\hline Batik Kawung & 290.3876 \\
\hline Batik Nitik & 271.2368 \\
\hline Batik Parang & 283.9238 \\
\hline Average & 287.9385 \\
\hline Pass Rate & $10 / 13$ \\
\hline
\end{tabular}

Table 10. Decryption measurements

\begin{tabular}{|c|c|c|c|}
\hline Image & BER & PSNR & SSIM \\
\hline Airplane gray & 0 & $\infty$ & 1 \\
\hline Baboon gray & 0 & $\infty$ & 1 \\
\hline House gray & 0 & $\infty$ & 1 \\
\hline Lena gray & 0 & $\infty$ & 1 \\
\hline Peppers gray & 0 & $\infty$ & 1 \\
\hline Airplane RGB & 0 & $\infty$ & 1 \\
\hline Baboon RGB & 0 & $\infty$ & 1 \\
\hline House RGB & 0 & $\infty$ & 1 \\
\hline Lena RGB & 0 & $\infty$ & 1 \\
\hline Peppers RGB & 0 & $\infty$ & 1 \\
\hline Batik Kawung & 0 & $\infty$ & 1 \\
\hline Batik Nitik & 0 & $\infty$ & 1 \\
\hline Batik Parang & 0 & $\infty$ & 1 \\
\hline Pass Rate & & $13 / 13$ & \\
\hline
\end{tabular}

square value, while the other 3 have not yet obtained the ideal chi-square value. However, the value of the three images has a chi-square value which is not too far from the ideal, so it can be concluded that this method has satisfactory results based on the chisquare.

The last test is the image decryption process, the aim is to determine the performance of the decryption process. The encrypted image must be completely decrypted so as not to change the meaning of the image. Therefore, to find out the decrypted image needs to be measured by bit error ratio (BER), peak signal to noise ratio (PSNR), and structural similarity index (SSIM). These three measuring instruments are widely used in various image security methods, such as cryptography and steganography $[5,36]$.

$$
\begin{array}{r}
B E R=\frac{\sum_{z=1}^{O} d_{z}}{M \times N \times 8} \\
d_{z}\left\{\begin{array}{l}
D_{z} \neq P_{z}, 1 \\
D_{z}=P_{z}, 0
\end{array}\right.
\end{array}
$$

BER serves to find out if there are errors that occur in the image bits, how to change the described image $(D)$ and plain image $(P)$ into binary form and reshape it into a 1-D array and then compare each bit at the same $z$ index, which is calculated with Eq. (13). The BER value must be 0 to get perfect decryption. While the PSNR is to find out how much noise occurs in the image encryption and decryption process, the value indicates that there is no noise in the described image. While SSIM is to check the image structure, if the value is 1 then the described image structure is the same as the plain image. These three measuring tools compare the plain image and the described image, where PSNR can be calculated by Eq. (14) and SSIM is calculated by Eq. (15).

$$
\begin{aligned}
& P S N R= \\
& 10 \log _{10}\left(\frac{255}{\frac{1}{M x N} \sum_{x}^{M} \sum_{y}^{N}[P(x, \mathrm{y})-D(x, \mathrm{y})]^{2}}\right) \\
& \\
& \quad \operatorname{SSIM~}(P, C)= \\
& \frac{\left(2 \mu_{P} \mu_{D}+v_{1}\right)\left(2 \sigma_{P D}+v_{2}\right)}{\left(\mu_{P}^{2}+\mu_{D}^{2}+v_{1}\right)\left(\sigma_{P}^{2}+\sigma_{D}^{2}+v_{2}\right)}
\end{aligned}
$$

Where $\mu_{P}$ is mean of the $P ; \mu_{D}$ is mean of the $D$; $\sigma_{P D}$ is the covariance $P$ against $D ; \sigma_{P}^{2}$ is a variant of $P ; \sigma_{D}^{2}$ is a variant of $D ; v_{1}=\left(l_{1} D\right)^{2}$ and $v_{2}=$ $\left(l_{2} D\right)^{2} ; D$ is a dynamic range $\left(2^{\text {bits }}-1\right)$ with the default value $l_{1}=0.01$ dan $l_{2}=0.03$

\section{Discussion}

In section 4 it can be seen that there are several comparisons of the methods presented. Comparison of color images and gray images. The comparison is done on the same dataset, namely the image of Lena. The previous encryption methods generally used a combination of diffusion and confusion methods. It can be seen that the proposed method is not entirely dominant, but after analyzing the results, it appears that the resulting method has very satisfactory values from various measurements. Based on statistical testing using the entropy value, all encrypted images have a value that is very close to the maximum value. The average value of entropy is very good, which is 7.9997. Meanwhile, for Lena's image with both 
grayscale and color types, the results look better than the previous methods, see Table 2 and Table 3, this means that the proposed method has a dominant contribution based on the entropy measurement tool. Other statistical measuring tools used are histogram and chi-square analysis. Visually based on the histogram, there was a significant histogram change besides the histogram pattern looked uniform in the bit distribution from 0 to 255 . Based on the chi-square value, three images still did not pass, but the average chi-square value of the 13 images showed that the histogram is relatively uniform.

Based on the differential attack test using UACI, NPCR, and AE measurements. It can be seen that this method is very superior because the average value of UACI and NPCR produced is very close to the ideal value $(\mathrm{UACI}=33.4635, \mathrm{NPCR}=99.6093)$, even when compared to the previous method in both grayscale and color images, especially in the Lena image. The proposed method looks superior, see Table 5 and Table 6. Meanwhile, the AE value is also very good because the average $\mathrm{AE}$ value is close to the ideal value $(50 \%)$.

At the decryption stage, the proposed method is proven to be able to carry out a perfect decryption process. This is evidenced by all images capable of producing a $\mathrm{BER}=0$, which indicates that there is no error in the bit value of the image, PSNR $=\infty$ which means that there is no noise at all and, SSIM $=1$ which means that there is no change in the image structure of the entire image after going through the encryption and decryption process.

These results prove that the proposed method produces a relatively better performance than the previous method. This is caused by gradual encryption and is performed in each local area of the image. In the initial encryption, each image area is encrypted with the same method but with a dynamic key based on the plain image hashing function, in the next stage, an XOR operation is carried out based on the logistic map and keyC of this section which increases the strength of statistical attacks. In addition, the hashing function on the key also increases the strength of the differential attack as evidenced by the UACI and NPCR values.

\section{Conclusions}

This study proposes an encryption model that combines two chaos methods with two hash functions. The first chaos method is carried out with zoning and rotation techniques based on key hash functions and plain text to create local encryption for each image zone. Furthermore, the overall encryption is carried out on the image with a logistic map. This encryption model uses the confusion process with the chaotic system in each image zone in the first step and the diffusion process in the second chaotic system is carried out thoroughly. Gradual encryption techniques have been shown to have a positive effect, so that this method can produce encrypted images that are resistant to statistical and differential attacks as evidenced by the measurement results of the average entropy $=7.9997, \mathrm{NPCR}=99.6085, \mathrm{UACI}=$ 33.4455 , avalanche effect $=50.0202$, relatively very uniform histogram, and chi-square analysis which has a pass rate of 10/13. In addition, all decryption processes can run perfectly as evidenced by measurements of $\mathrm{BER}=0, \mathrm{PSNR}=\infty$, and $\mathrm{SSIM}=1$. In the future, this method can certainly be developed to be even stronger, for example by using more key combinations, as well as more complex combinations of chaotic systems. However, it is also necessary to measure the efficiency of the method against computational costs.

\section{Conflicts of interest}

We wish to confirm that there are no known conflicts of interest associated with this publication and there has been no significant financial support for this work that could have influenced its outcome. We confirm also that the manuscript has been read and approved by all named authors and that there are no other persons who satisfied the criteria for authorship but are not listed.

\section{References}

[1] A. Setyono and D. R. I. M. Setiadi, "An Image Watermarking Method Using Discrete Tchebichef Transform and Singular Value Decomposition Based on Chaos Embedding", International Journal of Intelligent Engineering and Systems, Vol. 13, No. 2, pp. 140-150, 2020, doi: 10.22266/ijies2020.0430.14.

[2] X. Zhang, L. Wang, G. Cui, and Y. Niu, "Entropy-Based Block Scrambling Image Encryption Using DES Structure and Chaotic Systems", Int. J. Opt., 2019, doi: 10.1155/2019/3594534.

[3] F. Budiman and D. R. I. M. Setiadi, "A Combination of Block-Based Chaos with Dynamic Iteration Pattern and Stream Cipher for Color Image Encryption", International Journal of Intelligent Engineering and Systems, Vol. 13, No. 6, pp. 132-141, 2020, doi: 10.22266/ijies2020.1231.12.

[4] S. Kandar, D. Chaudhuri, A. Bhattacharjee, and B. C. Dhara, "Image encryption using sequence generated by cyclic group", J. Inf. Secur. Appl., 
Vol. 44, pp. 117-129, 2019, doi: 10.1016/J.JISA.2018.12.003.

[5] A. Susanto, D. R. I. M. Setiadi, E. H. Rachmawanto, I. U. W. Mulyono, C. A. Sari, M. K. Sarker, and M. R. Sazal, "Triple layer image security using bit-shift, chaos, and stream encryption", Bull. Electr. Eng. Informatics, Vol. 9, No. 3, pp. 980-987, 2020, doi: 10.11591/eei.v9i3.2001.

[6] S. Koppu and M. Viswanatham, "2D Chaotic Map Based on 2D Adaptive Grey Wolf Algorithm for Ultra Sound Medical Image Security", International Journal of Intelligent Engineering and Systems, Vol. 10, No. 1, 2017, doi: 10.22266/ijies2017.0228.12.

[7] B. Harjo and D. R. I. M. Setiadi, "Improved Color Image Encryption using Hybrid Modulus Substitution Cipher and Chaotic Method", International Journal of Intelligent Engineering and Systems, Vol. 14, No. 2, pp. 157-165, 2021, doi: 10.22266/ijies2021.0430.14.

[8] A. P. Kari, A. H. Navin, A. M. Bidgoli, and M. Mirnia, "A novel multi-image cryptosystem based on weighted plain images and using combined chaotic maps", Multimed. Syst., Vol. 27, No. 5, pp. 907-925, 2021, doi: 10.1007/s00530-021-00772-y.

[9] R. Lan, J. He, S. Wang, T. Gu, and X. Luo, "Integrated chaotic systems for image encryption", Signal Processing, Vol. 147, pp. 133-145, 2018, doi: 10.1016/J.SIGPRO.2018.01.026.

[10] C. Chen, K. Sun, and S. He, "An improved image encryption algorithm with finite computing precision", Signal Processing, Vol. 168 , p. 107340, 2020, doi: 10.1016/j.sigpro.2019.107340.

[11] Y. Luo, X. Ouyang, J. Liu, and L. Cao, "An Image Encryption Method Based on Elliptic Curve Elgamal Encryption and Chaotic Systems", IEEE Access, Vol. 7, pp. 3850738522, 2019, doi: 10.1109/ACCESS.2019.2906052.

[12] L. Xu, Z. Li, J. Li, and W. Hua, "A novel bitlevel image encryption algorithm based on chaotic maps", Opt. Lasers Eng., Vol. 78, pp. 17-25, 2016, doi: 10.1016/J.OPTLASENG.2015.09.007.

[13] M. Kanafchian and B. F. Vajargah, "A Novel Image Encryption Scheme Based on Clifford Attractor and Noisy Logistic Map for Secure Transferring Images in Navy", Int. J. ENavigation Marit. Econ., Vol. 6, pp. 53-63, 2017, doi: 10.1016/J.ENAVI.2017.05.007.

[14] C. Fu, G. Y. Zhang, M. Zhu, Z. Chen, and W. M.
Lei, "A New Chaos-Based Color Image Encryption Scheme with an Efficient Substitution Keystream Generation Strategy", Secur. Commun. Networks, Vol. 2018, 2018, doi: 10.1155/2018/2708532.

[15] M. Ge and R. Ye, "A novel image encryption scheme based on 3D bit matrix and chaotic map with Markov properties", Egypt. Informatics J., Vol. 20, No. 1, pp. 45-54, 2019, doi: 10.1016/J.EIJ.2018.10.001.

[16] C. Cao, K. Sun, and W. Liu, "A novel bit-level image encryption algorithm based on 2D-LICM hyperchaotic map", Signal Processing, Vol. 143, pp. 122-133, 2018, doi: 10.1016/J.SIGPRO.2017.08.020.

[17] M. Naim, A. A. Pacha, and C. Serief, "A novel satellite image encryption algorithm based on hyperchaotic systems and Josephus problem", Adv. Sp. Res., Vol. 67, No. 7, pp. 2077-2103, 2021, doi: 10.1016/J.ASR.2021.01.018.

[18] Z. Li, C. Peng, W. Tan, and L. Li, "An Effective Chaos-Based Image Encryption Scheme Using Imitating Jigsaw Method", Complexity, Vol. 2021, 2021, doi: 10.1155/2021/8824915.

[19] X. Wang, X. Zhu, X. Wu, and Y. Zhang, "Image encryption algorithm based on multiple mixed hash functions and cyclic shift", Opt. Lasers Eng., Vol. 107, pp. 370-379, 2018, doi: 10.1016/J.OPTLASENG.2017.06.015.

[20] D. Wei and M. Jiang, "A fast image encryption algorithm based on parallel compressive sensing and DNA sequence", Optik (Stuttg)., Vol. 238, p. 166748, 2021, doi: 10.1016/J.IJLEO.2021.166748.

[21] J. I. M. Bezerra, V. V. D. A. Camargo, and A. Molter, "A new efficient permutation-diffusion encryption algorithm based on a chaotic map", Chaos, Solitons \& Fractals, Vol. 151, p. 111235, 2021, doi: 10.1016/J.CHAOS.2021.111235.

[22] X. Wang and Y. Chen, "A New Chaotic Image Encryption Algorithm Based on L-Shaped Method of Dynamic Block", Sens. Imaging, Vol. 22, No. 1, 2021, doi: 10.1007/s11220-02100357-z.

[23] H. Diab, “An Efficient Chaotic Image Cryptosystem Based on Simultaneous Permutation and Diffusion Operations", IEEE Access, Vol. 6, pp. 42227-42244, 2018, doi: 10.1109/ACCESS.2018.2858839.

[24] F. Budiman, A. Suhendra, D. Agushinta, and A. Tarigan, "Determination of SVM-RBF kernel space parameter to optimize accuracy value of Indonesian Batik images classification", $J$. Comput. Sci., Vol. 13, No. 11, pp. 590-599, 2017, doi: 10.3844/jcssp.2017.590.599. 
[25] F. Budiman, "SVM-RBF parameters testing optimization using cross validation and grid search to improve multiclass classification", Sci. Vis., Vol. 11, No. 1, pp. 80-90, 2019, doi: 10.26583/sv.11.1.07.

[26] X. Wang and H. Sun, "A chaotic image encryption algorithm based on improved Joseph traversal and cyclic shift function", Opt. Laser Technol., Vol. 122, p. 105854, 2020, doi: 10.1016/j.optlastec.2019.105854.

[27] X. L. Chai, Z. H. Gan, Y. Lu, M. H. Zhang, and Y. R. Chen, "A novel color image encryption algorithm based on genetic recombination and the four-dimensional memristive hyperchaotic system - IOPscience", Chinese Phys. B, Vol. 25, 2016.

[28] D. Sravanthi, K. A. K. Patro, B. Acharya, and S. Majumder, "A secure chaotic image encryption based on bit-plane operation", in Advances in Intelligent Systems and Computing, Vol. 758, pp. 717-726, 2018.

[29] A. U. Rehman, X. Liao, R. Ashraf, S. Ullah, and $\mathrm{H}$. Wang, "A color image encryption technique using exclusive-OR with DNA complementary rules based on chaos theory and SHA-2", Optik (Stuttg)., Vol. 159, pp. 348-367, 2018, doi: 10.1016/j.ijleo.2018.01.064.

[30] Y. Li, C. Wang, and H. Chen, "A hyper-chaosbased image encryption algorithm using pixellevel permutation and bit-level permutation", Opt. Lasers Eng., Vol. 90, pp. 238-246, 2017, doi: 10.1016/j.optlaseng.2016.10.020.

[31] A. Babaei, H. Motameni, and R. Enayatifar, "A new permutation-diffusion-based image encryption technique using cellular automata and DNA sequence", Optik (Stuttg)., Vol. 203, p. 164000, 2020, doi: 10.1016/j.ijleo.2019.164000.

[32] S. Mortajez, M. Tahmasbi, J. Zarei, and A. Jamshidnezhad, "A novel chaotic encryption scheme based on efficient secret keys and confusion technique for confidential of DICOM images", Informatics Med. Unlocked, Vol. 20, p. 100396, 2020, doi: 10.1016/j.imu.2020.100396.

[33] Y. Jain, R. Bansal, G. Sharma, B. Kumar, and S. Gupta, "Image Encryption Schemes: A Complete Survey", Int. J. Signal Process., Vol. 9, No. 7, pp. 157-192, 2016, doi: 10.14257/ijsip.2016.9.7.15.

[34] A. Bakhshandeh and Z. Eslami, "An authenticated image encryption scheme based on chaotic maps and memory cellular automata", Opt. Lasers Eng., Vol. 51, No. 6, pp. 665-673, 2013, doi: 10.1016/j.optlaseng.2013.01.001.

[35] S. M. Seyedzadeh and S. Mirzakuchaki, "A fast color image encryption algorithm based on coupled two-dimensional piecewise chaotic map", Signal Processing, Vol. 92, No. 5, pp. 1202-1215, 2012, doi: 10.1016/j.sigpro.2011.11.004.

[36] D. R. I. M. Setiadi, "PSNR vs SSIM: imperceptibility quality assessment for image steganography", Multimed. Tools Appl., Vol. 80, No. 6, pp. 8423-8444, 2021, doi: 10.1007/s11042-020-10035-z. 